Kompass

Autoimmun

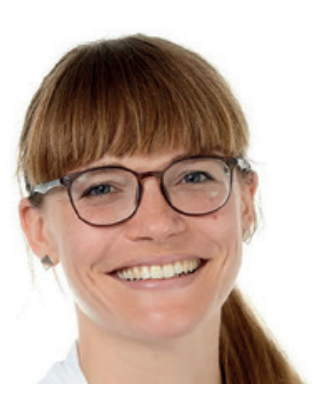

\title{
Das Kataraktrisiko für junge Typ-1-Diabetiker: Ergebnisse einer nationalen Kohortenstudie in Taiwan
}

Vita Dingerkus

Augenklinik, Stadtspital Triemli, Zürich, Schweiz

Abstract aus Lu WL, Shen PC, Lee CH, et al.: High risk of early cataracts in young type 1 diabetes group: a nationwide cohort study. Int J Endocrinol 2020;2020:8160256.

\begin{abstract}
Background: Chronic hyperglycemia in type 1 diabetes (T1D) patients results in ocular problems over time, but only a few studies emphasized on cataracts.

Aim: To evaluate the epidemiology of cataracts in the T1D population.

Method: A two-part study was conducted using data from the National Health Insurance Research Database in Taiwan. Information from the Longitudinal Health Insurance Database (LHID) was served as a template of the general population. In the first part, a total of 3,622 T1D cases registered between 1998 and 2007 were enrolled and compared with a matched group from the LHID. For identifying risk factors of cataracts in the T1D population in the second part, a total of 9032 T1D cases registered between 1998 and 2013 were included.
\end{abstract}

Results: Compared to the LHID, the hazard ratio (HR) of cataracts in the T1D group was 5.81 (95\% Cl 4.60-7.33), and the HR was higher in females $(6.29,95 \% \mathrm{Cl} 4.63-8.55)$. The peak incidence of cataracts occurred between age 20 and 29 in the T1D group, while in the LHID, it was after 60 . The overall incidence of cataracts in the T1D group was 9.1\%. In T1D patients with cataracts, they were found with higher rates of associated diabetic complications.

Conclusion: Compared to the nondiabetic population, cataracts seemed more rampant and premature in T1D patients, especially those of female gender. Early ophthalmologic examination should be considered in T1D patients.

(c) 2020 Wen-Li Lu et al. 


\section{Transfer in die Praxis}

\section{Hintergrund}

Die Katarakt ist eine bekannte, jedoch oft unterschätzte Komplikation bei Typ-1-Diabetikern. Der Aldose-Reduktase-Signalweg wird als Hauptursache für die diabetische Katarakt angenommen. Die Akkumulation von Sorbitol führt über erhöhten osmotischen Druck zur Schädigung der Linsenproteine und somit zur Linsentrübung [1]. Man geht von weiteren Faktoren aus, z.B. oxidativer Stress, autoimmune und genetische Faktoren.

Ziel der vorliegenden Studie ist es, epidemiologische Klarheit hinsichtlich Prävalenz und Risikofaktoren (RF) der diabetischen Katarakt zu schaffen, da deren Häufigkeit bei Typ-1-Diabetes (T1D) insbesondere im jungen Patientenalter im Vergleich zur diabetischen Retinopathie möglicherweise zu wenig Beachtung findet [2].

\section{Ergebnisse der Studie}

Die Studie besteht aus 2 Anteilen mit Patientendaten, die aus der taiwanischen «National Insurance Research Database» (NHIRD) und der «Longitudinal Health Insurance Database» (LHID) gespeist wurden. Einerseits wurden in den Jahren 1998-2007 3622 registrierte Patienten mit T1D für mindestens 5 Jahre beobachtet. Solche mit Computertomografie (CT)-Untersuchungen und bereits bekannter Katarakt wurden ausgeschlossen. Endpunkt war das Auftreten einer Katarakt im Vergleich zur gleich großen, auf Alter und Geschlecht abgestimmten Kontrollgruppe. Andererseits wurden Daten von 9032 registrierten Patienten aus den Jahren 1998-2013 auf folgende RF hin ausgewertet: Geschlecht, Auftreten von Retinopathie, Amputationen von Gliedern, Nierenerkrankung im Endstadium und Steroidtherapie. Statistische Methoden waren der ungepaarte T-Test für parametrische Daten und der Kaplan-Meier-Test für das Nichtvorhandensein von Katarakt mit einer Signifikanzmessung durch den Log-Rank-Test.

Die Hazard Ratio (HR) beim Vergleich beider Gruppen hinsichtlich des Auftretens von Katarakt lag bei 5,81. Bei der Gruppe der 20-29-Jährigen lag die höchste HR $(112,6)$ mit 101 Katarakten $(2,8 \%)$ in der T1D-Gruppe versus 1 Katarakt (0,2\%) in der Kontrollgruppe. Ebenfalls 101 Katarakte wurden unter den 10-19-jährigen Patienten mit T1D diagnostiziert. Unter den Patienten, bei denen die Diagnose eines T1D vor dem 10. Lebensjahr gestellt wurde, wurde mit einer durchschnittlichen Latenzzeit von 73,51 Monaten bei 17 Patienten $(0,5 \%)$ eine Katarakt diagnostiziert.

Die höchste Inzidenz bei der T1D-Gruppe lag zwischen dem 20. und 30. Lebensjahr. Während bei dieser die Inzidenz ab dem 30 Lebensjahr wieder abfiel, zeigte sich eine stetig ansteigende Inzidenz der Katarakt in der Kontrollgruppe mit fortschreitendem Alter. Um die RF für die Kataraktentwicklung näher einzugrenzen, wurde die zweite Datenanalyse in Hinblick auf die genannten Faktoren unter T1D-Patienten durchgeführt. Die Gruppe mit Katarakt wies ein signifikant höheres Vorkommen an Komplikationen des T1D auf. Ferner fand sich ein signifikanter Unterschied hinsichtlich des Geschlechts mit einer kumulativ höheren Inzidenz der Katarakt unter Frauen mit T1D, möglicherweise aufgrund des vermehrten Vorkommens an Aldose-Reduktase bei Frauen.
Systemischer Steroidgebrauch war signifikant seltener unter den an Katarakt leidenden Patienten.

\section{Fazit für die Praxis}

Die Stärke der Studie liegt vor allem in der großen Kohorte dank der großen Datenbank im taiwanischen Versicherungssystem. Auf einzelne Mängel gehen die Autoren größtenteils selbst in der Diskussion ein. Auffällig ist das Resultat hinsichtlich des Steroidgebrauchs in der Vorgeschichte. Zu kritisieren ist, dass die Autoren nicht von Beginn an alle Patienten mit Steroidgebrauch vom ersten Teil der Studie ausgeschlossen haben. Dass Steroidgebrauch nicht als RF in der zweiten Analyse identifiziert werden konnte, erklären sich die Autoren damit, dass dies bei der Entwicklung der diabetischen Katarakt eine untergeordnete Rolle spielen könnte. Wieso aber ist mit hoher Signifikanz der Steroidgebrauch häufiger unter T1D-Patienten ohne als bei solchen mit Kataraktentwicklung? Hier besteht Klärungsbedarf.

Dass auf den Hämoglobin-A1C (HbA1C)-Wert aufgrund fehlender Daten in der NHIRD nicht eingegangen wurde, rechtfertigen die Autoren mit ihrer Berücksichtigung anderer Spätfolgen des Diabetes. Dass jedoch unter Einbezug des HbA1C-Wertes eine detailliertere Risikoeinschätzung möglich und sinnvoll wäre, bevor der Patient fortgeschrittene Komplikationen erleidet, erscheint offensichtlich.

Zu Recht empfehlen die Autoren, dass auf Grundlage ihrer sowie vorgängiger Studien das Screening hinsichtlich der Kataraktentwicklung, insbesondere bei Kindern und Adoleszenten, früher, und zwar unmittelbar nach Erstdiagnose eines T1D, durchgeführt werden sollte [2].

Der Bundesärztekammer nach gilt derzeit die Empfehlung, dass «bei Typ-1-Diabetes ab dem elften Lebensjahr oder nach einer Diabeteserkrankungsdauer von fünf Jahren» [3] ophthalmologisch gescreent werden sollte, dies aufgrund des Risikos der Entwicklung der Retinopathie etwa 5 Jahre nach Erstdiagnose bei T1D [4]. Diese Empfehlung könnte sich aufgrund der verbesserten Datenlage hinsichtlich des Kataraktrisikos in nächster Zeit ändern.

\section{Disclosure Statement}

Bezüglich dieses Wissenstransfers bestehen keine Interessenkonflikte.

\section{Literatur}

1 Obrosova IG, Chung SSM, Kador PF: Diabetic cataracts: mechanisms and management. Diabetes Metab Res Rev 2010;26:172-180.

2 Simunović M, Paradžik M, Skrabić R, et al.: Cataract as early ocular complication in children and adolescents with type 1 diabetes mellitus. Int J Endocrinol 2018;2018:6.

3 https://www.leitlinien.de/mdb/downloads/nvl/diabetes-mellitus/dmnetzhautkomplikationen-2aufl-vers2-kurz.pdf.

4 Hooper P, Boucher MC, Cruess A, et al.: Canadian Ophthalmological Society evidence-based clinical practice guidelines for the management of diabetic retinopathy - executive summary. Can J Ophthalmol 2012;47:91-101.

\section{Korrespondenz an:}

Dr. Vita Dingerkus, Vita.Dingerkus@zuerich.ch 